\title{
The Dynamics of the Discrete Ultimatum Game and the Role of the Expectation Level
}

\author{
Lili Deng, ${ }^{1,2}$ Wei Zhang, ${ }^{1}$ Cheng Wang, ${ }^{3}$ Yi Han, ${ }^{2}$ and Jianhu Cai ${ }^{2}$ \\ ${ }^{1}$ Institute of Systems Engineering, Tianjin University, Tianjin 300072, China \\ ${ }^{2}$ College of Economics and Management, Zhejiang University of Technology, Hangzhou 310023, China \\ ${ }^{3}$ Institute of Industrial Engineering, Zhejiang University of Technology, Hangzhou 310014, China
}

Correspondence should be addressed to Cheng Wang; cwang@zjut.edu.cn

Received 21 January 2016; Accepted 12 April 2016

Academic Editor: Francisco R. Villatoro

Copyright (C) 2016 Lili Deng et al. This is an open access article distributed under the Creative Commons Attribution License, which permits unrestricted use, distribution, and reproduction in any medium, provided the original work is properly cited.

\begin{abstract}
We have studied evolutionary ultimatum game with spatially arranged players, who have choice between the two kinds of strategies (named greedy and altruist). The strategies in the ultimatum game here are described by $p(i)$ and $a(i)$, that is, the probability of offering $i$ to himself and the accepting probability when receiving $i$. By using computer simulations with $\mathrm{C}++$ builder, we have provided the dynamics of the greedy and altruistic strategies and found that the proportion evolution of the "greedy" strategy for different initial cases is approximately $60 \%$. Furthermore, the explanations for the interesting phenomenon are presented from different aspects. In addition, we illustrate that the factor of the expectation level (aspiration level) in the updating rule plays an important role in the promotion of altruistic behaviors.
\end{abstract}

\section{Introduction}

The issues of altruism and selfishness are in the centre of some of the most fundamental questions concerning our evolutionary origins, our social relations, and the organization of society. The investigation for the altruism is of great interest across biology and social sciences $[1,2]$. Moreover, experimental evidence indicates that human altruism is a powerful force in the animal world [3]. The ultimatum game is such a prime showpiece of the altruistic behaviors, while the rule of the ultimatum game is quite simple, which reads that two players are asked to divide a certain sum of money. One of the players, the proposer, suggests how to divide it, and the other player, the responder, has two choices: one is to agree to the division; the other one is to reject and thus both get nothing.

For the division strategy of the ultimatum game, the past decades have witnessed many theoretical investigations [46]. Many factors have been found to influence the outcomes of the ultimatum game, such as mutation [7], background payoff [8], payoff-oriented mechanism [9], degree-based assignation of roles [10], role preference [11], stochastic evolutionary dynamics [12], and the empathy mechanism [13]. Several studies focus on analyzing many types of connectivity structures. For instance, investigations on square lattice [7], small-world networks [14], scale-free networks [15], and adaptive networks $[16,17]$ have been conducted to primarily clarify the possible role of the topology.

Most of the abovementioned works have treated the strategies of the ultimatum game as continuous ones. But it can lead to some different and interesting outcomes by considering the strategies as discrete ones [6]. Nowak et al. [4] studied the four strategies of a minigame: $G_{1}=(h, l)$, $G_{2}=(l, l), G_{3}=(l, h)$, and $G_{4}=(h, h)$, where $l$ represents the low amount, while $h$ represents the high amout. They found that the ration $G_{1}=(h, l)$ dominated fairness $G_{3}=(l, h)$ in the stochastic case; while introducing the "reputation" factor, the fairness was favored. Szolnoki et al. [18] introduced a spatial ultimatum game with a discrete set of strategies and showed that this simple alteration could lead to fascinatingly rich dynamical behaviors. They [19] further illustrated the importance of discrete strategies in the ultimatum game 


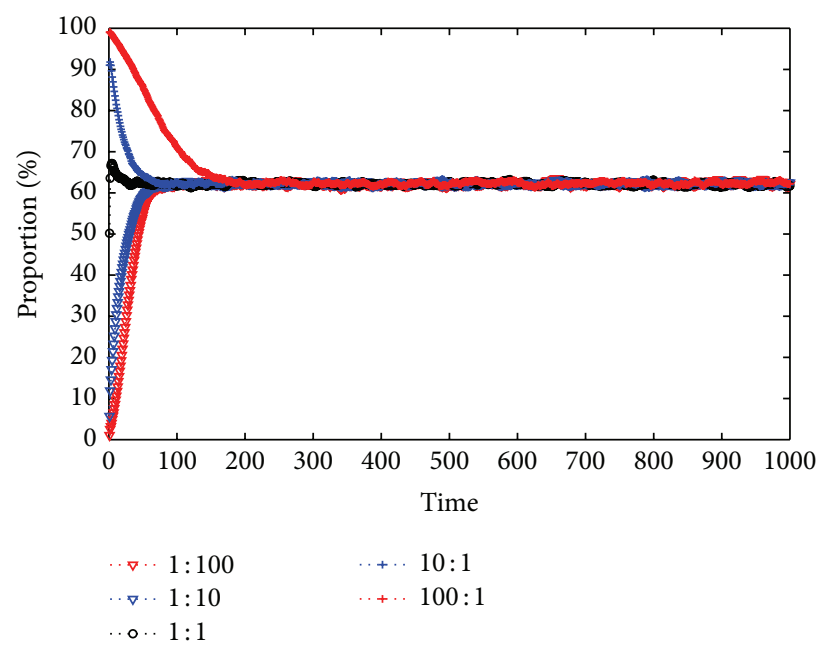

FIGURE 1: The proportion evolution of the "greedy" strategy for the five different initial cases.

and found that fine-grained strategy intervals promote the evolution of fairness in the spatial ultimatum game.

From a psychological perspective, models of social preferences [20] have been provided to formally explain the apparently irrational behavior [21] in the ultimatum game. The typical theories of negative reciprocity [22] focus on the intentions and describe rejection as a tool to punish the unfair proposer. The theories of inequality aversion [23] focus on the outcomes and claim that people are naturally averse to unequal distributions, especially when disadvantageous. By taking the prospect idea into considerations, Chen and Wang [24] introduced appropriate payoff aspirations in a smallworld networked game and found its profound effect on the promotion for cooperation. Perc and Wang [25] also showed that heterogeneous aspirations promoted cooperation in the Prisoner's Dilemma game.

Motivated by the above considerations, we follow [26] and adopt the two strategies to study their evolution in the spatial lattices $[27,28]$. These two strategies, that is, greedy and altruist, to some extent have some emotional significance, especially from the emotional perspectives. Furthermore, by introducing the expectation level into the updating rule, we will focus on the role of the expectation level for the altruist.

The outline of this paper is as follows. Section 2 shows the evolution of the greedy and altruistic strategies, from the viewpoints of the payoff, the type links, and the transform probabilities for illustration. Subsequently, by adding the expectation level, the evolution of altruist is given in Section 3. Finally, our conclusions are drawn in Section 4.

\section{Evolution of Greedy and Altruist Strategies}

The rule for the ultimatum game has been described above. Suppose that the total sum is $w$, and in the simulations the value for $w$ is set to be equal to 100. And the players participating in the game have the equal opportunity to be proposer or responder.

The strategy is generally denoted by $S=(p, a)$, where $p$ represents the amount given to the proposer per se and thus
TABLE 1: The descriptions for greedy and altruist strategies.

\begin{tabular}{lcc}
\hline Strategy & $p(i)$ & $a(i)$ \\
\hline Greedy & $2(i+1) /(w+1)(w+2)$ & $(i+1) /(w+1)$ \\
\hline Altruist & $2(w-i+1) /(w+1)(w+2)$ & 1 \\
\hline
\end{tabular}

the amount $w-p$ offered to the responder and the corresponding acceptance probability for the responder is denoted by $a$. Following [26], here, we consider the two particular forms for $(p, a)$ in the square lattices and the strategies are described as probability distributions:

(i) Greedy strategy: higher values are more probable to be accepted but lower amount is more probable to be proposed to others.

(ii) Altruist strategy: higher values are more probable to be proposed to others.

The quantitative description for the two strategies is provided in Table 1 , where $i \in\{0,1,2, \ldots, w\}$.

We first study the spatial and the temporal evolution of the two strategies in the square lattices. Firstly, each player is denoted by one site in two-dimensional square lattices, and this system size $L=n \times n$ in simulations has been set to be $L=100 \times 100$. Initially, each site is occupied by any one of the two strategies. In each round of games, each player plays the game with its immediate eight neighbors in the square lattice with periodic boundary. The score for each one is the sum of the payoff in these eight encounters. At the start of the next generation, each lattice-site is occupied either by one of the neighbors or by its previous owner, depending on who obtains the highest payoff in that round and so to next round $[27,28]$. One run of the model consists of 1000 generations. Each experimental condition is replicated 30 runs and all the data, other than the snap, is the average of these 30 runs.

The average proportion of "greedy" strategy in the population only with "greedy" and "altruist" strategy is shown in Figure 1, with the different ratios of greedy sites 


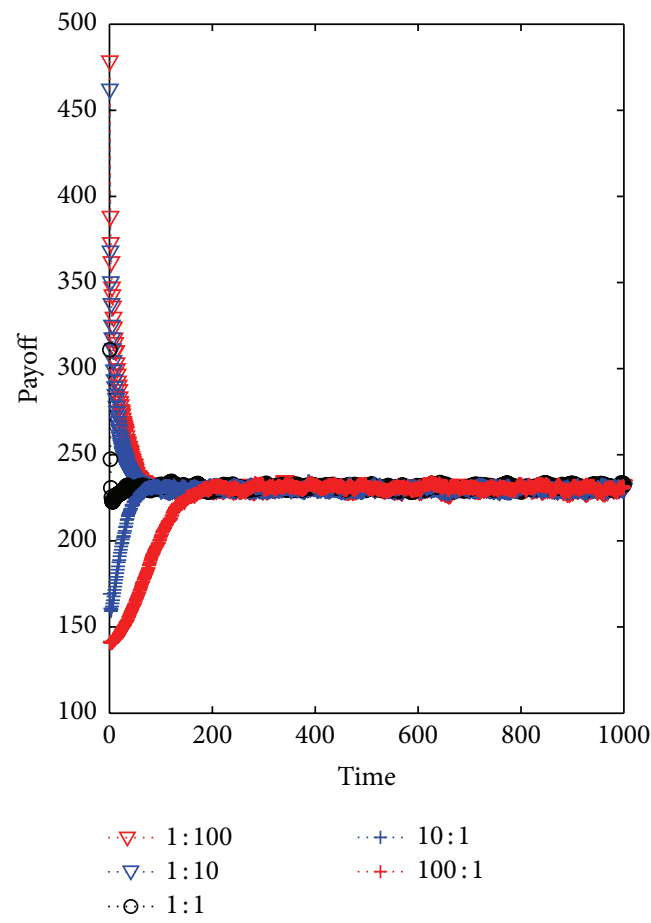

(a)

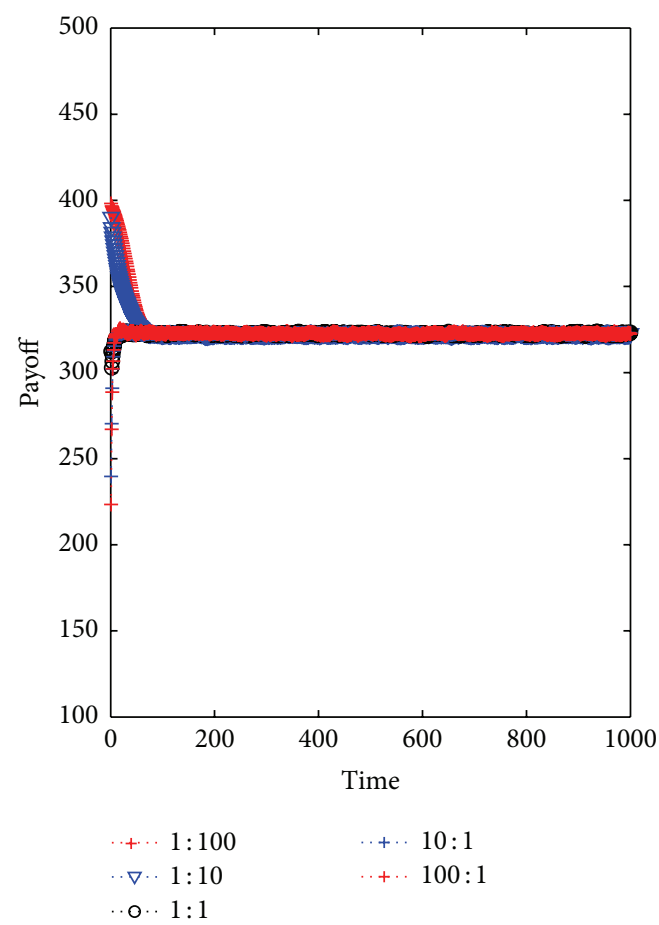

(b)

FIgURE 2: The evolution for payoff of (a) greedy and (b) altruist.

(G) : altruist ones (A): $1: 100,1: 10,1: 1,10: 1$, and $100: 1$, respectively. From Figure 1, it is obvious that the final situations are quite similar and the "greedy" population is about 60 percent in the whole population, almost independent of the initial conditions. The result of the simulation reveals that the "altruist" and "greedy" strategies could coexist.

Combining with the average payoff for greedy population and the altruist ones, shown in Figure 2, one can quantitatively compare that the payoff of the altruist (about 320 ) is much bigger than those of the greedy population (about 240). But the fraction of the greedy population (about $60 \%$ ) is bigger than that of altruist (about $40 \%$ ). Naturally, one may wonder what causes this to happen.

To give an intuitional illustration, we show the asymptotic pattern in Figure 3. Here, it is worth stating that the same asymptotic results arise with other initial cases. The color coding is as follows: yellow represents an altruist (A) site following $A$ in the preceding generation; aqua is a greedy $(G)$ site following G; red is A following G; and black is G following A. From Figure 3, one can easily see that yellow and aqua cluster together, while red and black lie in between yellow and aqua.

From the perspective of the type of links, Figure 4 further shows the evolution of different link types, where A-A denotes a link connecting nodes with both altruist strategies; G$\mathrm{G}$ represents a link connecting nodes with both greedy strategies; and A-G is a link connecting nodes with the two different strategies. We further show that the asymptotic results are the same for the different G:A ratios. Clearly, the asymptotic results show that, among all the links, the GG occupies the first place; A-A comes second; and A-G is the last.

The amounts of red and black in Figure 3 imply how many sites are varying from one generation to the next, which is correspondingly shown in Figure 5. Figure 5(a) shows the conditional probability, where $\operatorname{Pr}\left(S_{t}=\mathrm{G} \mid S_{t-1}=\mathrm{G}\right)+$ $\operatorname{Pr}\left(S_{t}=\mathrm{A} \mid S_{t-1}=\mathrm{G}\right)=1$ means that the $\mathrm{G}$ players in the preceding $(t-1)$ th generation either remain as $\mathrm{G}$ or change into $\mathrm{A}$ in the $t$ th generation and so to $\operatorname{Pr}\left(S_{t}=\mathrm{A} \mid S_{t-1}=\right.$ A) $+\operatorname{Pr}\left(S_{t}=\mathrm{G} \mid S_{t-1}=\mathrm{A}\right)=1$. Figure 5(b) directly shows the proportions of the four different colors at the $t$ th generation $(t=0,1,2, \ldots, 100)$. One can easily see that the proportions of $A \rightarrow G$ and $G \rightarrow A$ are the same during the asymptotic patterns after few generations.

\section{Expectation Effect on the Evolution of Altruist}

In this section, we proceed to the exploration of the greedy and altruist by introducing the expectation level. The strategy updating relies on the difference between player's actual payoff and the expectation level. Following previous works [24], we define a parameter $E \in[0, w]$ as the average expectation level of the players, and each player calculates its expectation payoff $P_{E}=k E$, where $k$ denotes the number of neighbors and in this square lattice each is identical as $k=8$. During the evolutionary process, player $j$ will compare the sum of payoff (denoted by $P_{j}$ ) from neighbors with 


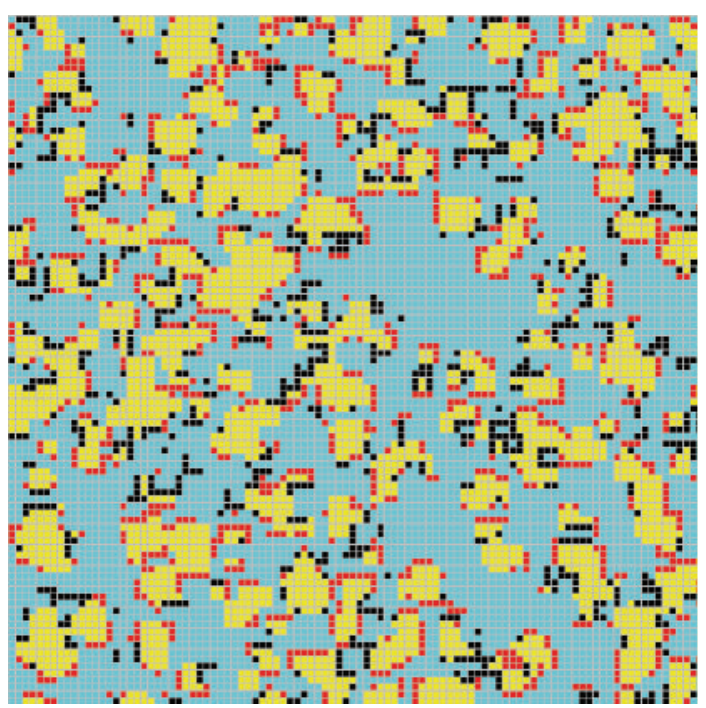

FIGURE 3: The snap after 20 generations for the greedy $(\mathrm{G})$ and altruist (A) strategies, initially with $50 \%$ altruist and $50 \%$ greedy players.

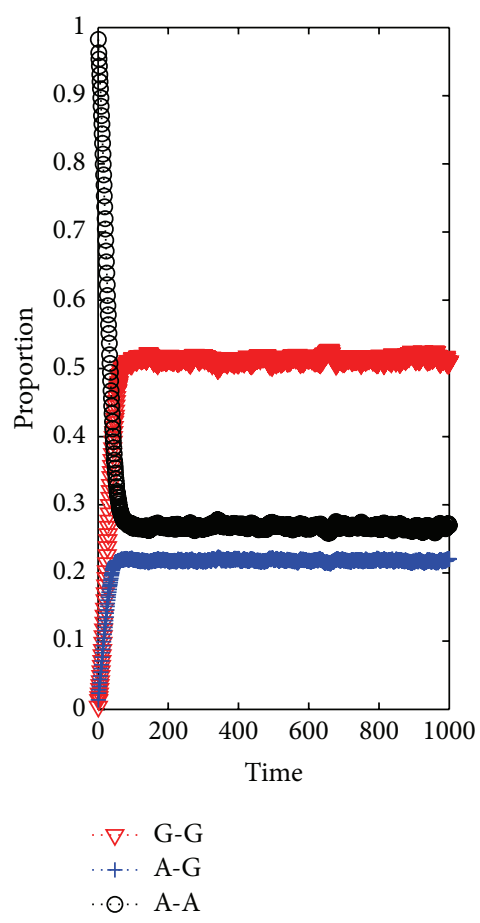

(a)

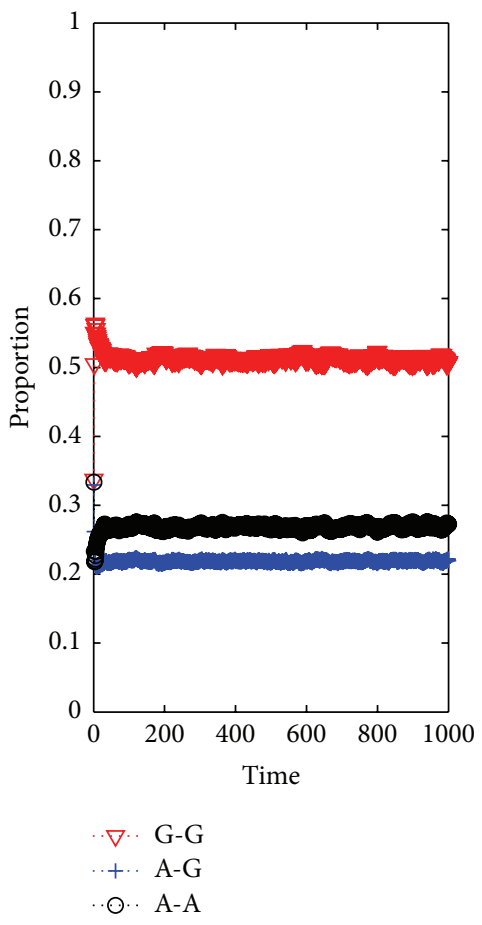

(b)

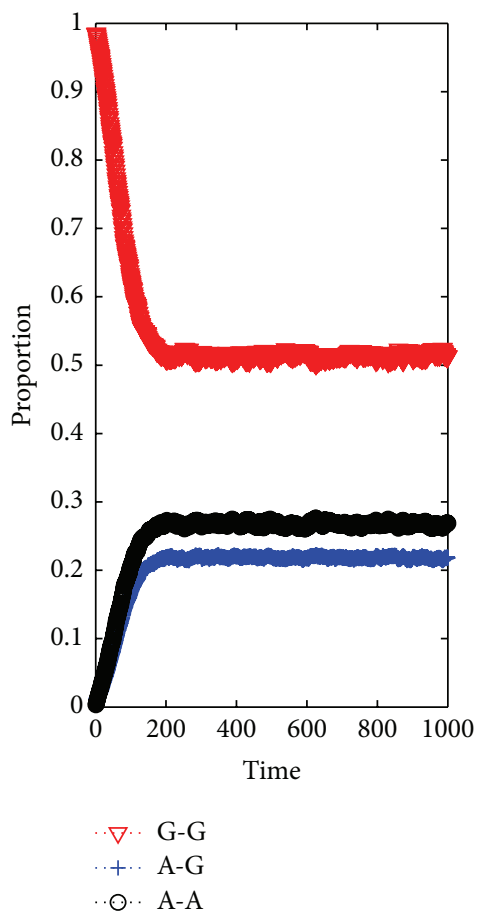

(c)

Figure 4: The evolution for the proportion of links (G-G, G-A, and A-A) with initially different G: A ratios (a) $1: 100$, (b) $1: 1$, and (c) $100: 1$.

the expectation level $P_{j E}$ and change its current strategy to its opposite strategy with a probability depending on the difference $\left(P_{j}-P_{j E}\right)$ as

$$
W_{j}=\frac{1}{1+\exp \left[\left(P_{j}-P_{j E}\right) / \kappa\right]},
$$

where $\kappa$ characterizes the noise effects in the strategy adoption process. The aspiration level $P_{j E}$ provides the benchmark which is used to evaluate whether player $j$ satisfies its current strategy. This evolutionary rule is stochastic. The rationale is that players can make use of their own payoff information efficiently and evaluate their satisfaction levels toward their current strategies more accurately. This probability characterizes the exact extent of changing their current strategies. Herein, we simply set $\kappa=1$ in this section and concentrate on how the expectation payoff affects the evolution of altruist on this square lattice. 


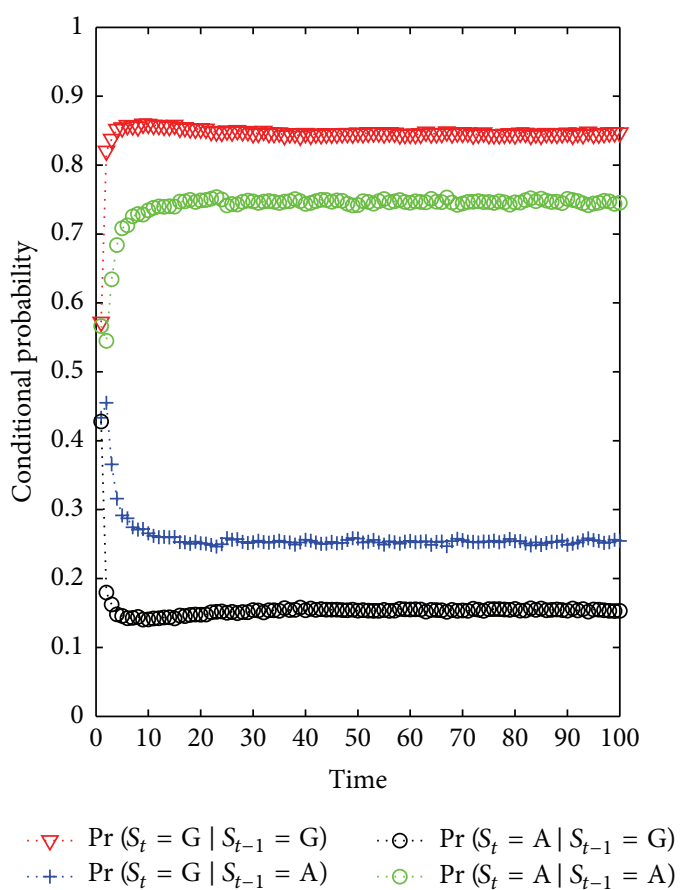

(a)

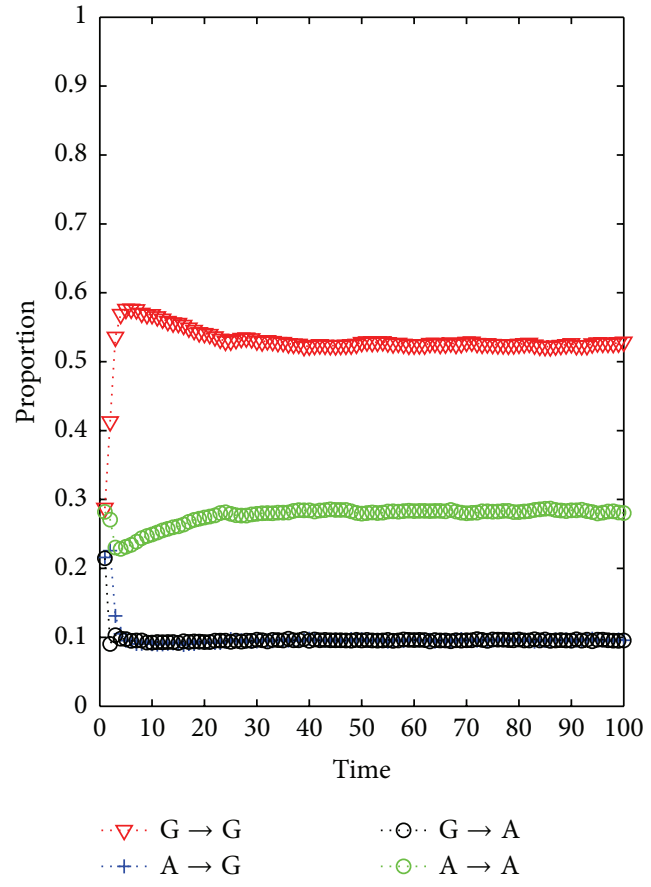

(b)

FIGURE 5: The (a) conditional probability and the (b) proportion of the strategy changing.

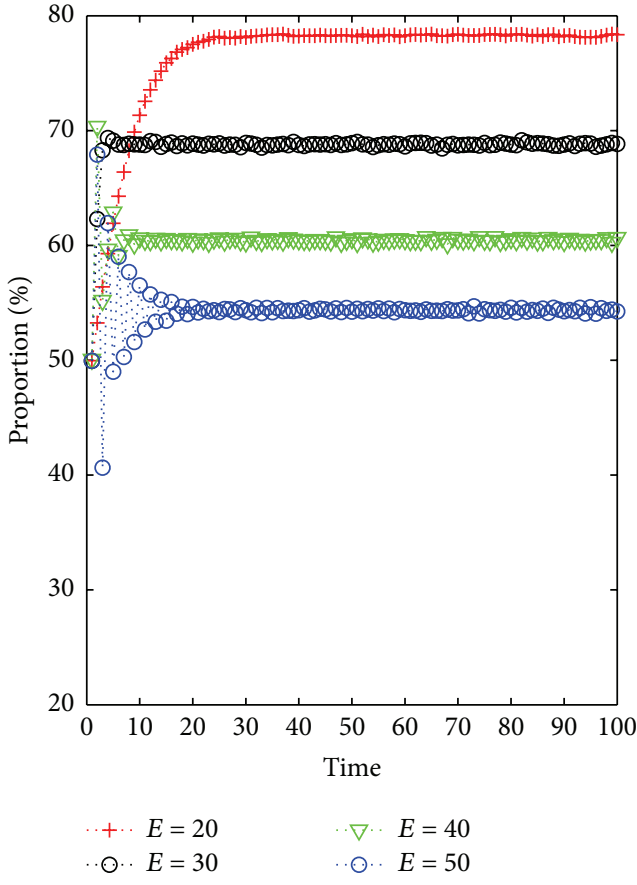

(a)

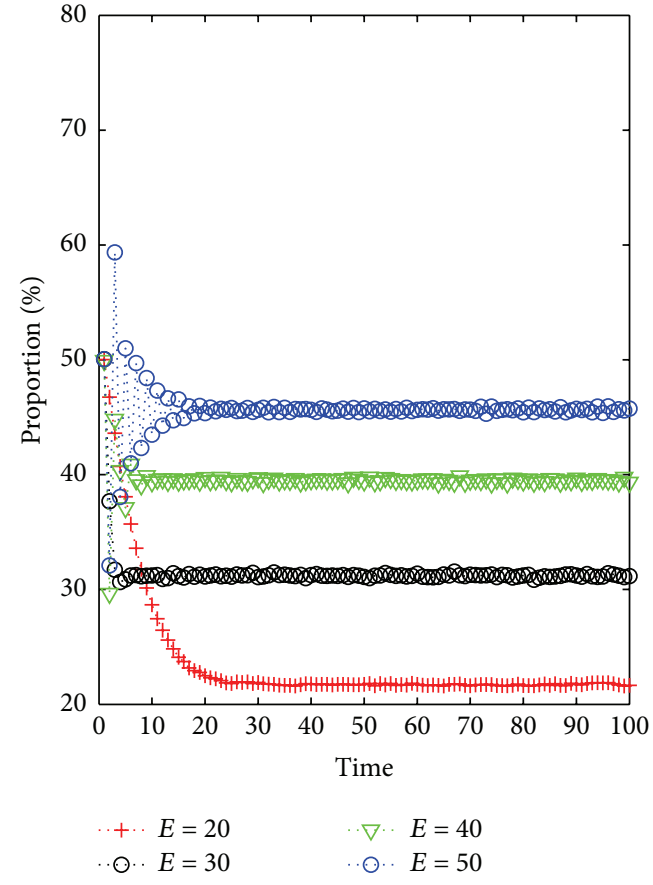

(b)

FIgURE 6: The proportion for (a) greedy and (b) altruist.

From Figure 6, one can see that the evolution of altruist to some extent is related to the expectation level. Figure 6(b) clearly shows the altruist fraction increases with $E$. The tendency is quite similar to that of payoff shown in Figure 7.
Figure 8 shows the evolution of strategy types for different $E$. Similarly, after few generations $(t<20)$, the proportion of $A \rightarrow G$ is identical with that of $G \rightarrow A$. With increasing $E$, the fractions of $\mathrm{G} \rightarrow \mathrm{G}$ and $\mathrm{A} \rightarrow \mathrm{A}$ are reduced, while 


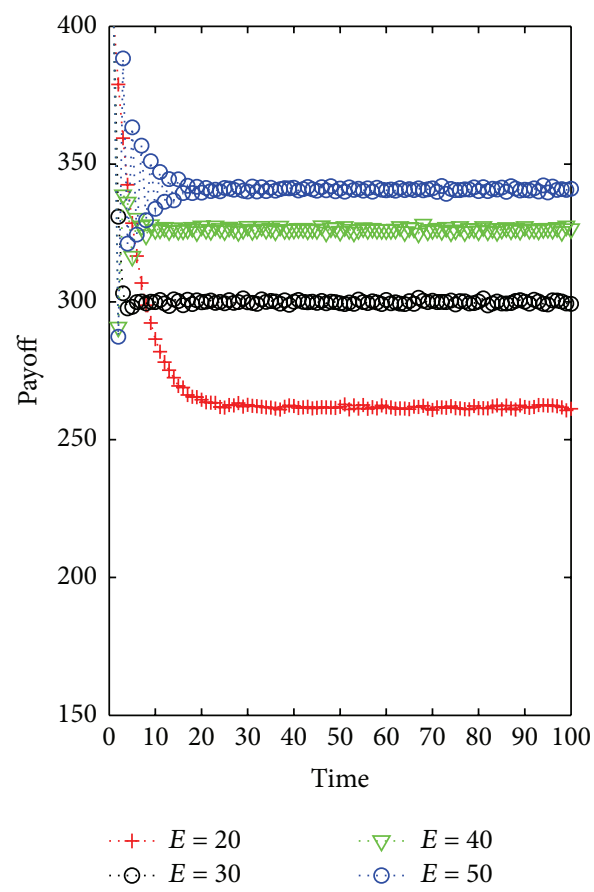

(a)

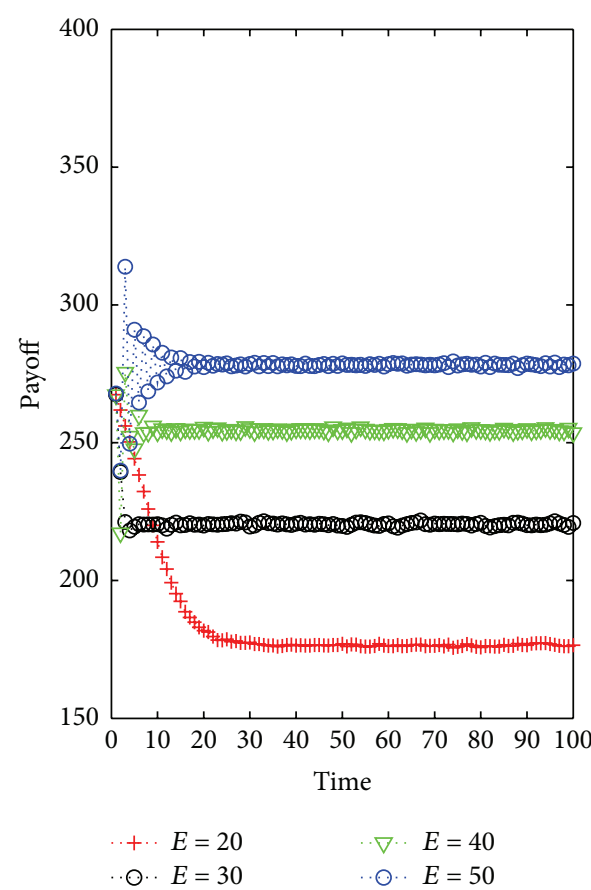

(b)

FIGURE 7: The payoff for (a) greedy and (b) altruist.

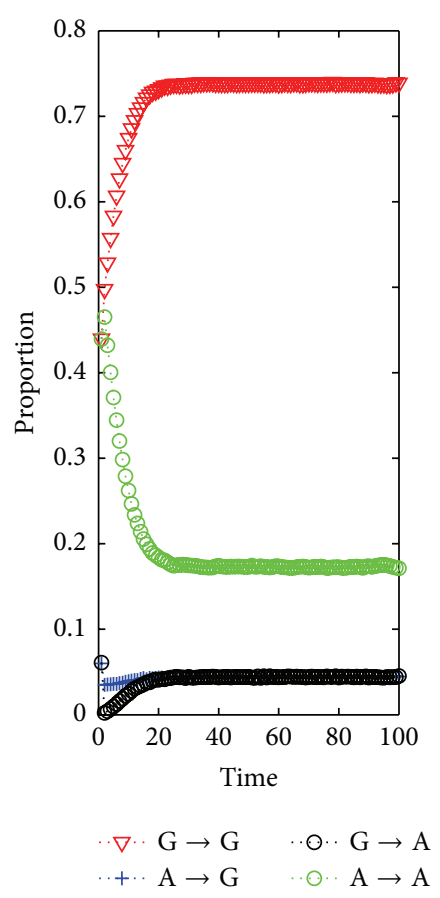

(a)

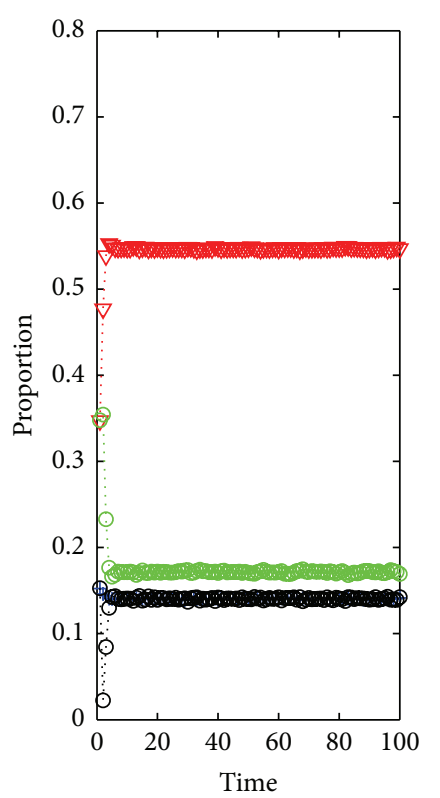

$\nabla \cdot \mathrm{G} \rightarrow \mathrm{G} \quad \cdot \odot \cdot \mathrm{G} \rightarrow \mathrm{A}$

.. $\cdot \mathrm{A} \rightarrow \mathrm{G}$

(b)

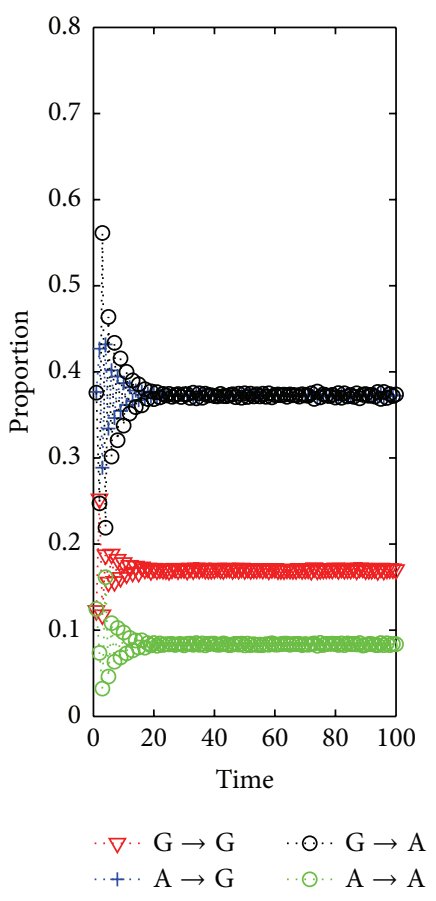

(c)

Figure 8: The proportion of strategy changing: (a) $E=20$, (b) $E=30$, and (c) $E=50$.

the fractions of $\mathrm{A} \rightarrow \mathrm{G}$ and $\mathrm{G} \rightarrow \mathrm{A}$ increase simultaneously. Intuitively speaking, it is quite reasonable that the higher the expectation, the bigger the difference between expectation and the actual payoff, and thus the higher the probability changing.
As for the strategy pairs, Figure 9 shows the fraction that the player with $G(A)$ connecting with $G(A)$ or A has $G$ neighbor. As $E$ increases, both A-A link and A-G link increase, while G-G link decreases. This thus implies that expectation promotes altruist. 


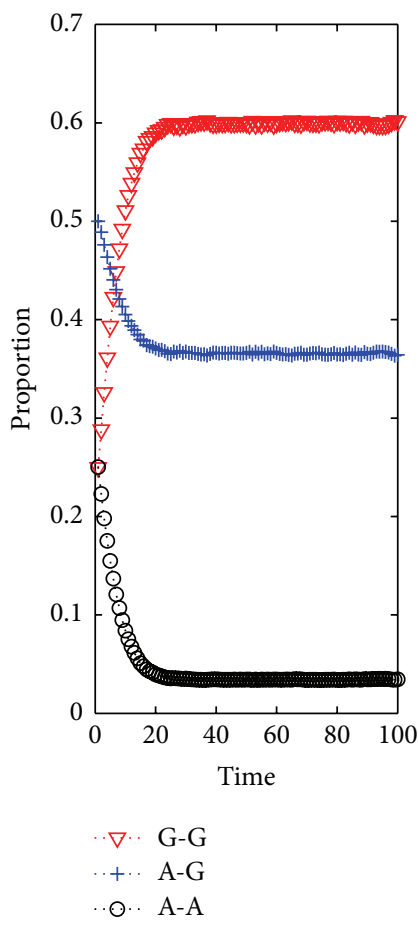

(a)

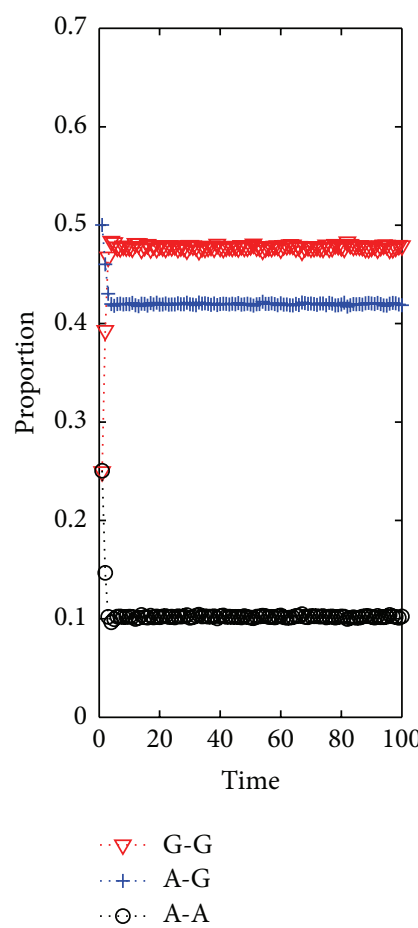

(b)

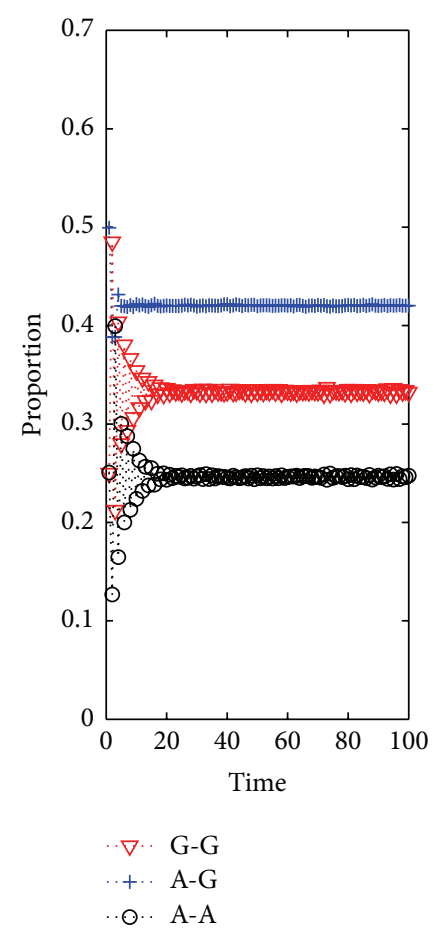

(c)

FIgURE 9: The proportion evolution of link: (a) $E=20$, (b) $E=30$, and (c) $E=50$.

\section{Conclusion}

The competition between the "greedy" and "altruist" strategies is studied by numerical simulations and the results of evolutionary processes are plotted for some relevant cases. From the perspectives of payoff evolution, snaps, the transform probability, and the link type, we give the intuitional illustrations. Furthermore, to make full use of one's own information, the expectation level is introduced into the strategy updating mechanism. The simulation results show that the expectation level can to some extent promote the fraction of altruist.

\section{Competing Interests}

The authors declare that they have no competing interests.

\section{Acknowledgments}

This work was supported partially by the National Natural Science Foundation of China Grant nos. 71401158, 71301148, 71371170,71301147 , and 71572184, partially by the Natural Science Foundation of Zhejiang Province Grant nos. LY15G010009 and LY16G010013, and partially by the Humanities and Social Science Foundation of Zhejiang University of Technology.

\section{References}

[1] S. Gao, T. Wu, S. Nie, and L. Wang, "Promote or hinder? The role of punishment in the emergence of cooperation," Journal of Theoretical Biology, vol. 386, pp. 69-77, 2015.
[2] J. C. Schank, P. E. Smaldino, and M. L. Miller, "Evolution of fairness in the dictator game by multilevel selection," Journal of Theoretical Biology, vol. 382, pp. 64-73, 2015.

[3] E. Fehr and U. Fischbacher, "The nature of human altruism," Nature, vol. 425, no. 6960, pp. 785-791, 2003.

[4] M. A. Nowak, K. M. Page, and K. Sigmund, "Fairness versus reason in the Ultimatum Game," Science, vol. 289, no. 5485, pp. 1773-1775, 2000.

[5] K. M. Page and M. A. Nowak, "A generalized adaptive dynamics framework can describe the evolutionary ultimatum game," Journal of Theoretical Biology, vol. 209, no. 2, pp. 173-179, 2001.

[6] M. Dishon-Berkovits and R. Berkovits, "The ultimatum game: discrete vs. continuous offers," Physica A: Statistical Mechanics and Its Applications, vol. 409, pp. 53-60, 2014.

[7] K. M. Page, M. A. Nowak, and K. Sigmund, "The spatial ultimatum game," Proceedings of the Royal Society of London, Series B: Biological Sciences, vol. 267, no. 1458, pp. 2177-2182, 2000.

[8] L. Deng, W. Tang, and J. Zhang, "The coevolutionary ultimatum game on different network topologies," Physica A: Statistical Mechanics and its Applications, vol. 390, no. 23-24, pp. 42274235, 2011.

[9] W.-Q. Duan and H. E. Stanley, "Fairness emergence from zerointelligence agents," Physical Review E, vol. 81, no. 2, Article ID 026104, 2010.

[10] Z. Li, J. Gao, I. H. Suh, and L. Wang, "Degree-based assignation of roles in ultimatum games on scale-free networks," Physica A: Statistical Mechanics and Its Applications, vol. 392, no. 8, pp. 1885-1893, 2013.

[11] G. Ichinose, "Coevolution of role preference and fairness in the ultimatum game," Complexity, vol. 18, no. 1, pp. 56-64, 2012.

[12] D. G. Rand, C. E. Tarnita, H. Ohtsuki, and M. A. Nowak, "Evolution of fairness in the one-shot anonymous ultimatum 
game," Proceedings of the National Academy of Sciences of the United States of America, vol. 110, no. 7, pp. 2581-2586, 2013.

[13] K. M. Page and M. A. Nowak, "Empathy leads to fairness," Bulletin of Mathematical Biology, vol. 64, no. 6, pp. 1101-1116, 2002.

[14] M. N. Kuperman and S. Risau-Gusman, "The effect of the topology on the spatial ultimatum game," The European Physical Journal B, vol. 62, no. 2, pp. 233-238, 2008.

[15] R. Sinatra, J. Iranzo, J. Gómez-Gardeñes, L. M. Floría, V. Latora, and Y. Moreno, "The ultimatum game in complex networks," Journal of Statistical Mechanics: Theory and Experiment, vol. 2009, no. 9, Article ID P09012, 2009.

[16] L.-L. Deng, W.-S. Tang, and J.-X. Zhang, "Coevolution of structure and strategy promoting fairness in the ultimatum game," Chinese Physics Letters, vol. 28, no. 7, Article ID 070204, 2011.

[17] L. L. Deng, C. Wang, W. S. Tang, G. G. Zhou, and J. H. Cai, "A network growth model based on the evolutionary ultimatum game," Journal of Statistical Mechanics: Theory and Experiment, vol. 2012, no. 11, Article ID P11013, 2012.

[18] A. Szolnoki, M. Perc, and G. Szabó, "Defense mechanisms of empathetic players in the spatial ultimatum game," Physical Review Letters, vol. 109, no. 7, Article ID 078701, 2012.

[19] A. Szolnoki, M. Perc, and G. Szabó, "Accuracy in strategy imitations promotes the evolution of fairness in the spatial ultimatum game," Europhysics Letters, vol. 100, no. 2, Article ID 28005, 2012.

[20] E. Halali, Y. Bereby-Meyer, and A. Ockenfels, "Is it all about the self? The effect of self-control depletion on ultimatum game proposers," Frontiers in Human Neuroscience, vol. 7, article 240, 2013.

[21] C. Civai, "Rejecting unfairness: emotion-driven reaction or cognitive heuristic?" Frontiers in Human Neuroscience, vol. 7, article 126, 2013.

[22] M. Rabin, "Incorporating fairness into game theory and economics," The American Economic Review, vol. 86, no. 5, pp. 12811303, 1993.

[23] E. Fehr and K. M. Schmidt, "A theory of fairness, competition, and cooperation," Quarterly Journal of Economics, vol. 114, no. 3, pp. 817-868, 1999.

[24] X. J. Chen and L. Wang, "Promotion of cooperation induced by appropriate payoff aspirations in a small-world networked game," Physical Review E, vol. 77, no. 1, Article ID 017103, 2008.

[25] M. Perc and Z. Wang, "Heterogeneous aspirations promote cooperation in the prisoner's dilemma game," PLoS ONE, vol. 5, no. 12, Article ID e15117, 2010.

[26] R. da Silva, G. A. Kellermann, and L. C. Lamb, "Statistical fluctuations in population bargaining in the ultimatum game: static and evolutionary aspects," Journal of Theoretical Biology, vol. 258, no. 2, pp. 208-218, 2009.

[27] M. A. Nowak and R. M. May, "Evolutionary games and spatial chaos," Nature, vol. 359, no. 6398, pp. 826-829, 1992.

[28] M. A. Nowak and R. M. May, "The spatial dilemmas of evolution," International Journal of Bifurcation and Chaos, vol. 3, no. 1, pp. 35-78, 1993. 


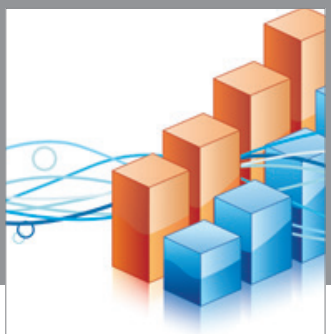

Advances in

Operations Research

vatem alat4

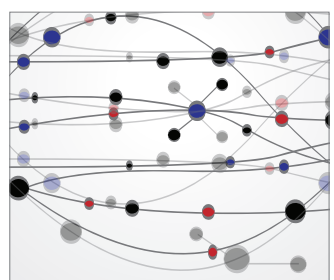

\section{The Scientific} World Journal
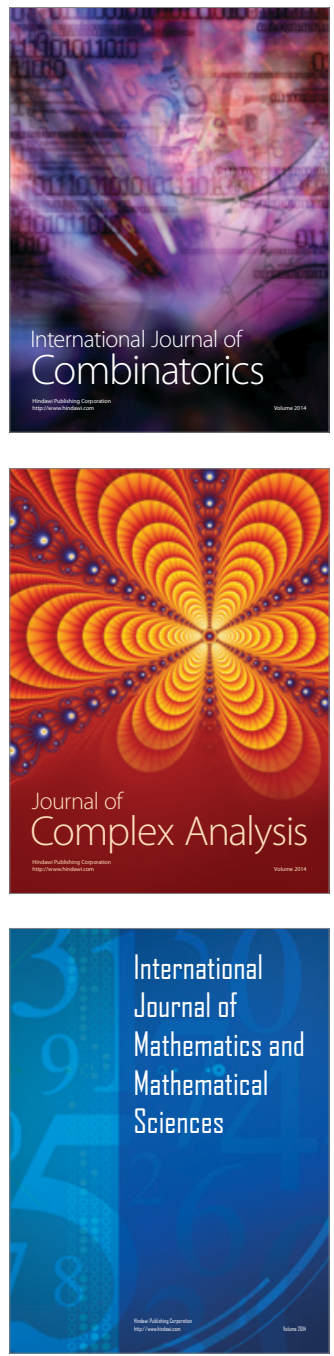
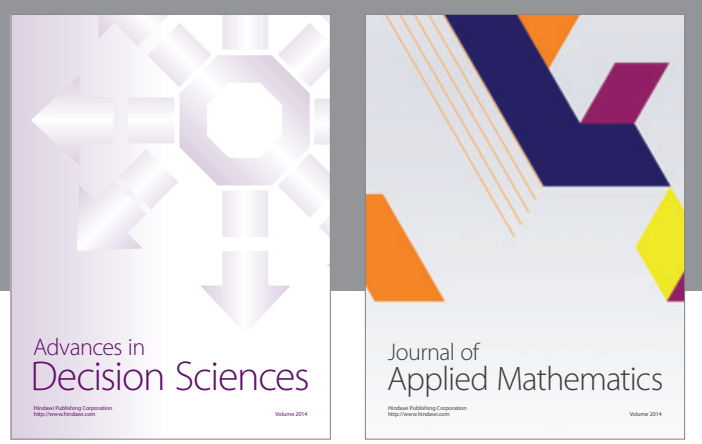

Algebra

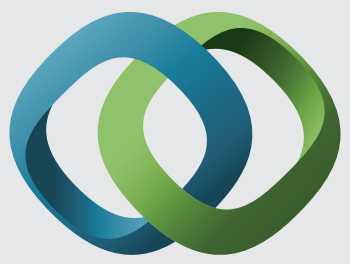

\section{Hindawi}

Submit your manuscripts at

http://www.hindawi.com
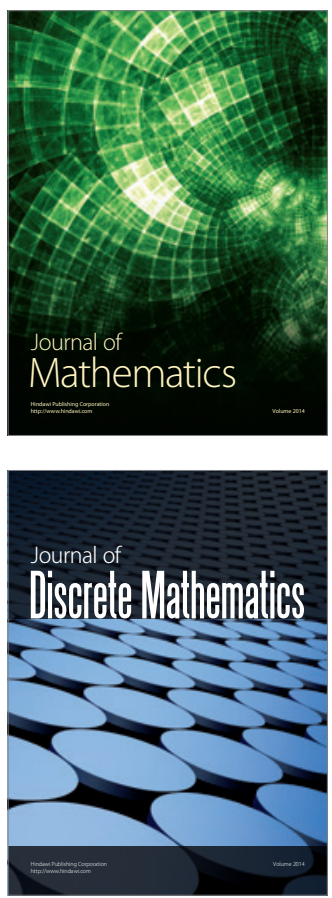

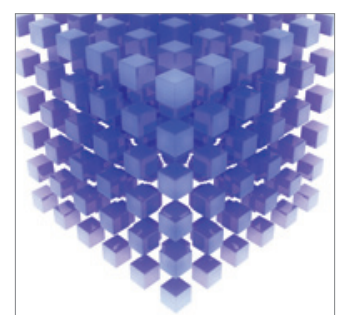

Mathematical Problems in Engineering
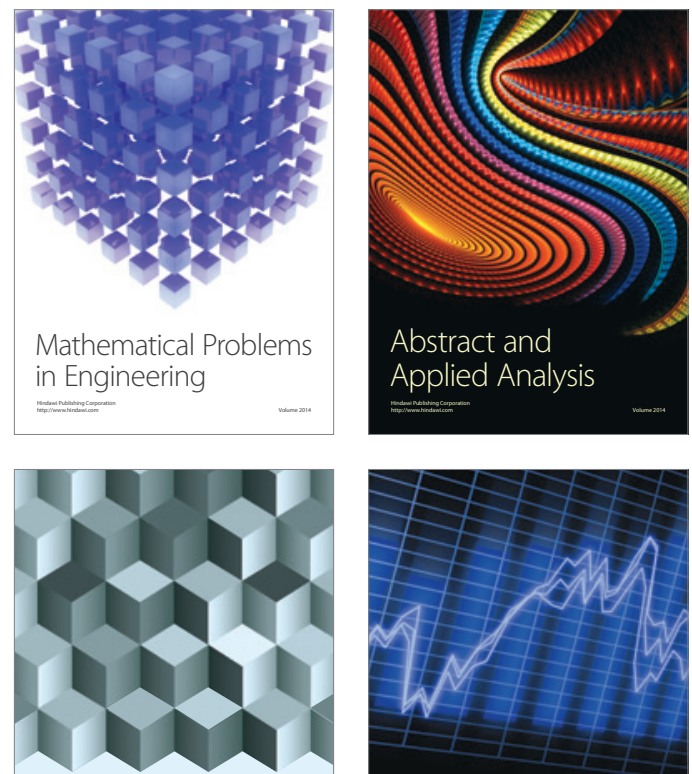

Journal of

Function Spaces

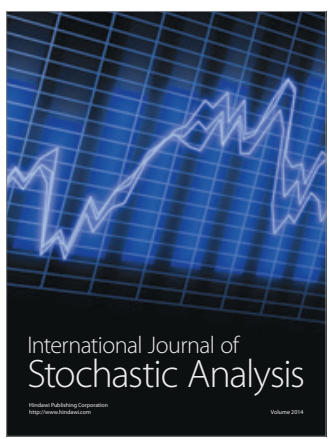

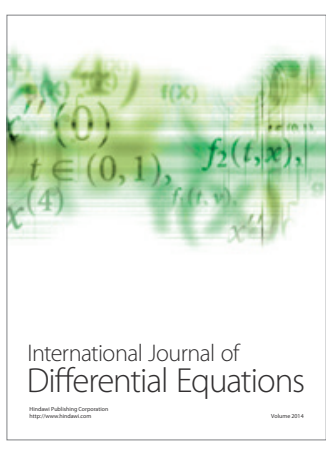
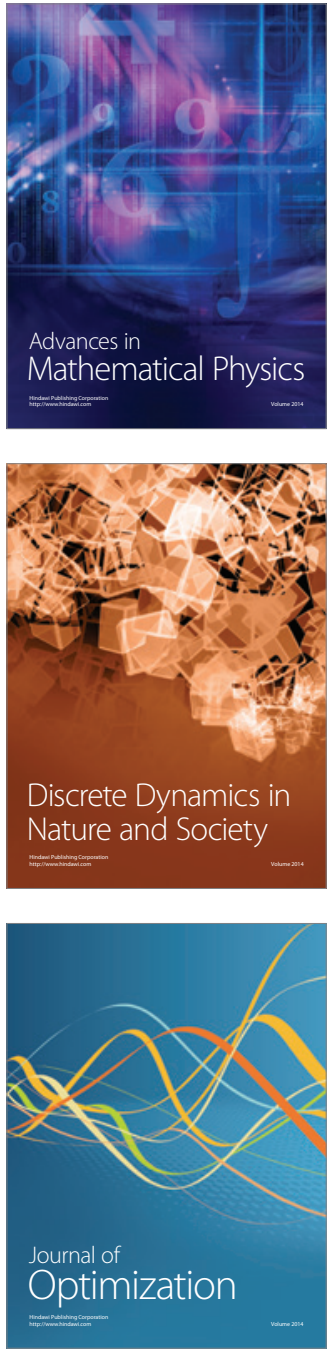